Portland State University

PDXScholar

Summer 2020

\title{
The Meaning of Red and Green in User Interfaces for the Color Deficient
}

Bassel Hamieh

Portland State University

Follow this and additional works at: https://pdxscholar.library.pdx.edu/honorstheses

Part of the Graphics and Human Computer Interfaces Commons Let us know how access to this document benefits you.

\section{Recommended Citation}

Hamieh, Bassel, "The Meaning of Red and Green in User Interfaces for the Color Deficient" (2020). University Honors Theses. Paper 924.

https://doi.org/10.15760/honors.946

This Thesis is brought to you for free and open access. It has been accepted for inclusion in University Honors Theses by an authorized administrator of PDXScholar. Please contact us if we can make this document more accessible: pdxscholar@pdx.edu. 
The Meaning of Red and Green in User Interfaces for the Color Deficient

\author{
by \\ Bassel Hamieh
}

An undergraduate honors thesis submitted in partial fulfillment of the

requirements for the degree of
in
University Honors
and
Computer Science

Thesis Adviser

Ellie Harmon

Portland State University

2020 


\title{
The Meaning of Red and Green in User Interfaces for the Color Deficient
}

\author{
Bassel Hamieh \\ Computer Science \\ Portland State University \\ bahamieh@pdx.edu
}

\begin{abstract}
Around 300 million people worldwide are colorblind which is a problem because one important way we communicate in digital interfaces is through the use of color. Red and green are two colors that are especially heavily used in interface design because of their strong symbolic associations: red being a sign to warn or stop and green being the opposite. This has a large effect on red-green colorblind people who are not able to perceive either of those colors correctly. Many solutions exist that aim to help users better differentiate colors, but none take into account color symbolism. In this project, I explore red and green color symbolism through interviews with four red-green deficient individuals. I identified five key findings relevant to interface designers: colorblindness varies from person to person, colorblind individuals already have many creative work arounds, colorblind people still understand color symbolism, charts and patterns are very difficult to understand, and current accessibility modifications alter the user experience. Based on these findings, I outline three implications for design: interfaces should retain meaningful colors, consistency is critical, and customization is ideal.
\end{abstract}

\section{CCS CONCEPTS}

Human-centered computing, Human computer interaction (HCI), Interaction design, Collaborative and social computing, Accessibility

\section{KEYWORDS}

Color deficiency, color blindness, Red-Green, Symbolism, Human computer interaction, Interface design

\section{INTRODUCTION}

Around $8 \%$ of males and $.5 \%$ of females around the world suffer from colour vision deficiency, also known as colorblindness, where $99 \%$ are red-green colorblind [1]. That is around 300 million people worldwide that do not perceive color the way the rest do. According to UX Planet, 108 million web users are colorblind, not counting colorblind individuals who only use smartphone and smart device interfaces [14]. This is a problem when a lot of the way we communicate, largely through technology, is with the use of color. Red is often used to represent danger, warnings, or errors, while green is used to symbolize the opposite, such as pass, good-to-go, and error free [4, 7].
Many attempts have been made to help colorblind individuals to better see colors, ranging from color changing algorithms to augmented reality [8]. However, such attempts often over-focus on color differentiation, and there is a lack of attention placed on the color symbology. In this paper, I examine how to build or adapt interfaces that are not only usable, but that also maintain the purpose and the symbolism behind intended designs.

Through a review of background information and related work, I show how important color symbolism is to interface design, and also how existing accessibility interventions and standards over-focus on color differentiation with a disregard for color symbolism. I then interviewed 4 colorblind individuals in order to develop answers to 2 key questions:

1. What might be lost for colorblind users when the problem is framed narrowly as a problem of contrast as opposed to more broadly a problem of communication?

2. Are there ways to design or adapt a digital interface that would make it not only technically accessible but also reflective of the symbolic associations connected to the colors red and green?

In this paper I present five key findings from these interviews: colorblindness varies from person to person, colorblind individuals already have many creative workarounds, colorblind people still understand color symbolism, charts and patterns are very difficult to understand, and current accessibility modifications alter the user experience. Based on these findings, I close with three implications for design: interfaces should retain meaningful colors, consistency is critical, and customization is ideal.

\section{BACKGROUND}

\section{The Facts}

Color blindness, specifically red-green colorblindness, is relatively common among men. The number of males with red-green colorblindness accounts for almost $8 \%$ of the entire world population, as compared to only about $0.5 \%$ of females [2]. It is mostly hereditary but can also be caused by injury [4]. It is among the most common color vision deficiencies inherited by children from their parents, and is passed from a recessive gene attached to a X-chromosome 
from the mother to the son. This affects the way the eye sees color [4]. The retina has light-sensitive cells called cones which are responsible for colour discrimination. The three types of cones are red, green and blue, which work together to help humans see the entire color spectrum. When a person is colorblind, it means that one or more of those cones aren't working correctly. Therefore, when an individual is red-green colorblind, it means their red and green cones are not behaving properly and therefore are not able to distinguish certain shades of red and green.

A person is determined to be colorblind through a vision exam based on Ishihara plates (see Figure 1), which is the most common form of testing done for the color deficient. The plates consist of colored dots in colors designed to test different types of colorblindness. They fill the entire plate and contain a number or a path [4]. People with normal color vision should be able to see the number or identify the path, while people with colorblindness should not.

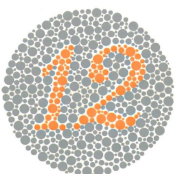

(a)

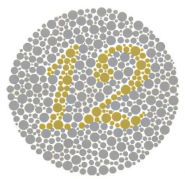

(d)

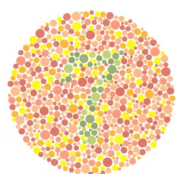

(b)

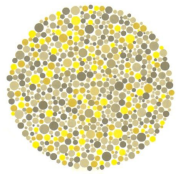

(e)

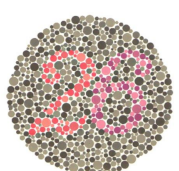

(c)

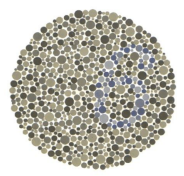

(f)
Figure 1. Ishihara test plates. (a-c) Plates as viewed by a normal color observer; (d-f) simulated versions of how they are seen by a red deficient person [6].

\section{A History of Color}

Red and green are naturally occurring colors in our environments. They are used to help humans communicate ideas based on the symbolisms associated with them, which have evolved over time. Red is one of the most vibrant colors on the color wheel and one of the ones with the richest history. Known to be the first color ever used, it has been seen in 40,000 year old prehistoric cave paintings, in Ancient Egyptian and Greek murals, in Byzantine Empire clothing, and in all art movements up until the modern day [3]. Red symbolizes an array of strong emotions and experiences such as love, passion, sexuality, anger, heatness, evil, and danger. In Asian cultures such as China and India, it symbolizes well being and good fortune, in Russia and neighboring cultures it symbolizes revolution and communism, in African cultures such as Nigeria it symbolizes aggression and death, and in western cultures such as the United States, it symbolizes passion, danger, and love [7]. But where did all of these symbolic meanings and interpretations that we attach to the color red come from? After all, red is simply a pigment extracted from materials such as iron-rich soils and at one point, insects.

The constructs behind the color red have evolved over time and there are a number of theories that try to explain the origins of current meanings. Red is often nature's warning sign as it exists on many venomous organism's bodies such as coral poison dart frogs, black widow spiders, and synanceia verrucosa stonefish [4]. This can be a reason why we currently associate red with a lot of our warning signs such as stop signs. Red is also the color of fire and fire is hot and dangerous. This association can be the reason we use red to express heat and caution. Red is also the color of our blood and organs, and when blood seeps out of the human body, it is not usually a good sign, which could have been the reason why it has historically been associated with danger, death, pain, and evil. While red has been known to represent extreme and complex emotions, green mostly evokes a more neutral and positive connotation.

Unlike red, green didn't have an early representation in prehistoric art since it was a very difficult pigment to reproduce and therefore was always represented later on as a dull and more brown-like green in ancient times. It was not until synthetic green was created in 1835 , that a more vibrant green began appearing in art [9]. Green has been seen early in history. Some examples are when the Ancient Egyptians used it to create paintings of Osiris, in Muslim architechture and iconography as it was seen as a sacred color, in Jan Van Eyck's iconic portrait "The Arnolfini Wedding", and all the way into the modern art movement [3]. Green symbolizes peace, renewal, growth, wealth, goodluck, and nature throughout history. In Ireland, green symbolises good luck. In western cultures green symbolizes money, jealousy, and also luck. In Latin America green symbolizes death. And in Asia, green symbolizes eternity, wealth, fertility, and sometimes infidelity [7]. The color green has garnered numerous constructus over time which can be due to many theories, with the strongest being that green is almost everywhere in nature. Nature naturally brings peace and grows abundantly. These associations can be the reasons why we think of nature, growth, wealth, and renewal when it comes to the color green and why we use it to represent those concepts.

\section{Red \& Green in Interface Design}

Seeing that red and green have developed very strong symbolic meanings by humans, it is clear why they have been used heavily as tools for communication and expression for centuries; and computer interface design is no exception to this fact. There is a heavy use of red and green in User Experience (UX), User Interface (UI), web, and application design. According to Babich, a User Experience professional and editor of the online magazine UX Planet, "both red and green are extremely important for UI design because they are actionable." In web design, red usually symbolizes importance, danger, warning, or an error state. And there is a very clear example to explain why: "When designers use red for delete buttons, the color naturally gives users pause due to its [historic] connotations." [14].

It is also used to evoke an emotional response and is associated with love and passion. A good example of this is the heart button on Instagram. Green, on the other hand, symbolizes growth, safety, a success state and generally a positive outcome. "An action which is complete, a file which has been saved or an online order which was placed" are all outcomes with associations to the color green [14]. Both green and red are used individually in UX design, but 
are more often used together; specifically for binary operations such as declining or accepting a phone call.

The very prominent uses of both colors in UX design can be extremely problematic for red-green color deficient individuals who have trouble distinguishing or seeing these colors properly [5]. Most people are not colorblind and therefore web designs often do not take into consideration color-blind users. This is why a good set of accessibility standards are important for all web designers to follow, in order to maximize the accessibility and effectiveness of their designs. As stated in UX Planet, it is important to not "rely on color alone to convey the meaning."

\section{RELATED WORK}

\section{Accessibility Standards}

The World Wide Web Consortium (W3C), an international standards body, has developed a set of Web Content Accessibility Guidelines (WCAG) for developers to follow in order to ensure accessibility $[13,16]$. These guidelines include recommendations about the use of color, and contrast which are applicable to creating web interfaces that are accessible to the color deficient population. A webpage that further details the Use of Color guideline, in particular, provides the following advice for colorblind friendly web content [11]:

1. Ensuring that information conveyed by color differences is also available in text

2. Including a text cue for colored form control labels

3. Ensuring that additional visual cues are available when text color differences are used to convey information

4. Using a contrast ratio of 3:1 with surrounding text and providing additional visual cues on focus for links or controls where color alone is used to identify them

5. Using color and pattern

6. Ensuring that information conveyed by color differences is also available in text.

Numerous tools for web designers are available to automatically check if a design meets some of the WCAG suggestions such as the contrast ratio standards [12]. In addition to these international accessibility standards for the colorblind, there are many researchers which have worked on interventions, methods, and tools, to help make the web and other interfaces more accessible for the color deficient $[1,15]$.

\section{Current Methods of Accessibility}

While there are countless attempts at aiding the color deficient when it comes to Human Computer Interaction, recoloring algorithms and augmented reality seem to be two of the most popular and innovative ones to date [10].

Recoloring algorithms attempt to automatically generate a version of a website or other digital interface that uses different colors. These algorithms are used for two main reasons: the first is to help designers see their work the way a color deficient person might see them; the second is to recolor images to help the colorblind more easily recognize and differentiate colors they would not have been able to see otherwise. Within these algorithms are three main requirements which must be met in order to create the most accurate coloring representations for both designers and the color deficient - color naturalness, color consistency, and color contrast [8]. Figure 3 shows an example of the results of one such image recoloring algorithm. Research shows that these algorithms both allow colorblind individuals to better differentiate colors when using web interfaces and also allow web designers to identify deficiencies in their designs as perceived by color deficient individuals, and as a result, create web content that is more accessible [8].

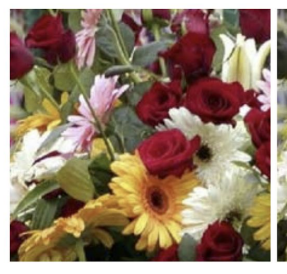

(a)

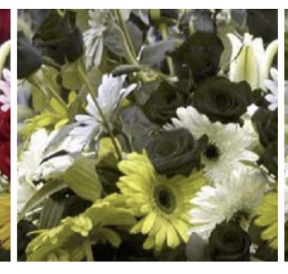

(b)

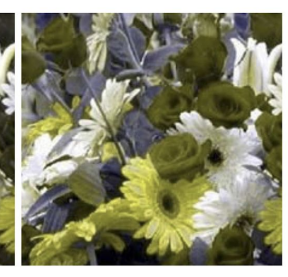

(c)
Figure 3. HSV-based recoloring method (a) original image; (b) simulated red deficient person image view; (c) red deficient image view after recoloring [8].

Augmented reality on the other hand is used to alleviate the obstacles that colorblind people face when identifying and differentiating colors during everyday tasks such as cooking, choosing outfits, reading signs and maps, creating art, and manipulating photos through interviews. One such intervention is Chroma, a wearable real-time augmented-reality application based on Google Glass, which promises to address real-life issues of colorblind people [10]. Chroma has many color blindness modes some of which are highlighting mode, contrast mode, daltonization, and outlining. In tests of the Chroma technology, colorblind participants were able to identify more colors correctly than before when doing mundane everyday things.

\section{CRITIQUE OF LITERATURE}

Many attempts have been made to help colorblind individuals to better see colors, ranging from setting international accessibility standards, to color changing algorithms, to augmented reality [8] and more. However, such attempts often over-focus on color differentiation, and there is a lack of attention placed on the color symbology. By intervening through the use of things such as color overlays or adding additional information channels (e.g., an icon), red and green's important symbolic dimensions in culture are ignored and color history, color theory, or the important meanings tied to red and green are forgotten. There isn't enough research which looks into maintaining the purpose and symbolism behind web designers' intended designs, explore how current interventions for red-green color deficient accessibility alter the meaning and intent of user interfaces, or examine what strategies can be used to maintain symbolic dimension of color, while also ensuring accessibility for the red-green color deficient. This research aims to fill this gap by exploring two questions:

1. What might be lost for colorblind users when the problem is framed narrowly as a problem of contrast as opposed to more broadly as a problem of communication? 
2. Are there ways to design or adapt a digital interface that would make it not only technically accessible but also reflective of the symbolic associations connected to the colors red and green?

\section{METHODS}

After conducting the above literature reviews to synthesize information on red-green color deficiency, the history of red and green, how the two are used in interface design, accessibility standards for the color deficient in the web interface arena, and the current methods of accessibility available to color deficient people, it was clear that the research space lacked an understanding of how the role of symbolisms and associations to colors fit into web interface communication.To gain a better perspective on how color symbology is perceived and affects the red-green color deficient community, a set of 4 interviews were conducted with red-green deficient individuals to learn about their experiences, challenges, and perceptions of color symbolism when experiencing real world user interfaces. This was done with the greater goal of exploring how to design or adapt a digital interface that is accessible and reflective of the symbology connected to the colors red and green.

\section{Participants and Interview Overview}

Recruitment for the interviews was done through social networks and the leveraging of a snowball sampling technique to recruit more participants. Once participants were identified, the interviews were conducted by video-conferencing through Portland State University 's Zoom Video Platform. They ranged from 33 to 68 minutes in length with an average of 48 minutes per interview and were split into three distinct sections with different purposes.

The first section of the interview consisted of a set of preliminary questions about the interviewee, their relationship with colorblindness, and their symbolic associations to the colors red and green.

The second section of the interview used sets of images depicting various digital interfaces to give interviewees the opportunity to reflect on their color experience in the context of real user interface examples. I used three different sets of images, and showed them to participants based on time available during the interview. The three image sets are discussed in the next sections.

The third and final section of the interview consisted of a set of in-depth questions about the interviewee's difficulties using web interfaces, what tools they use to manage those difficulties and their reasons behind them, what their ideal colorblind aid tool would look like, and lastly how their perception of color affects their interaction with web interfaces.

\section{Image Set 1}

The first image set consists of 6 mobile interface images, 3 of which are shown in Figures 4, 5, and 6. All images depicted variations of an interface for answering or declining an incoming call on a smartphone. I created each variation myself in order to examine whether the color deficient interviewee has the same preconceived notions of red and green as others who are not colorblind and to gain insights on what kind of information is needed to be present for a deficient individual to make informed decisions.

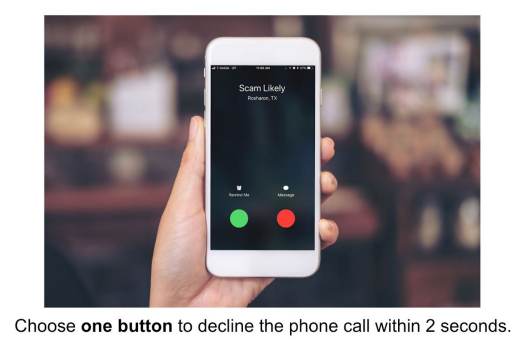

Figure 4. Mobile interface with a prompt to choose a button to decline the phone call within two seconds. The colors of the buttons are green (left) and red (right) with the iconography taken out. The buttons were switched.

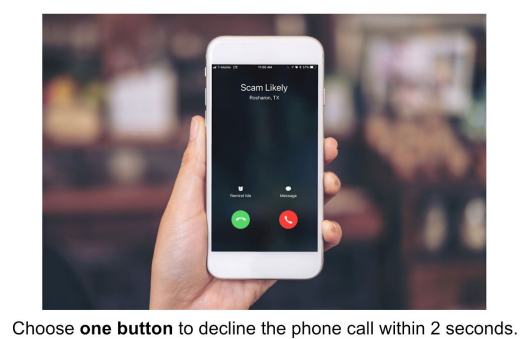

Figure 5. Mobile interface with a prompt to choose a button to decline the phone call within two seconds. The colors of the buttons are green (left) and red (right) with the iconography usually associated with those colors switched.

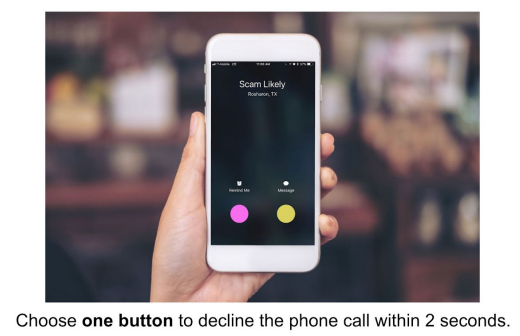

Figure 6. Mobile interface with a prompt to choose a button to decline the phone call within two seconds. The colors of the buttons are purple (left) and yellow (right) with the iconography taken out.

\section{Image Set 2}

The second set of images consists of 3 before and after slides of a mobile game interface with and without colorblind mode activated (one sample screenshot is shown in Figure 7). These were used to examine how a mobile game's colorblind mode affects it's playability, attractiveness, and difficulty level to the color deficient interviewee and to promote discussion about colorblind modes, whether they are helpful, and how they are perceived by colorblind individuals. 


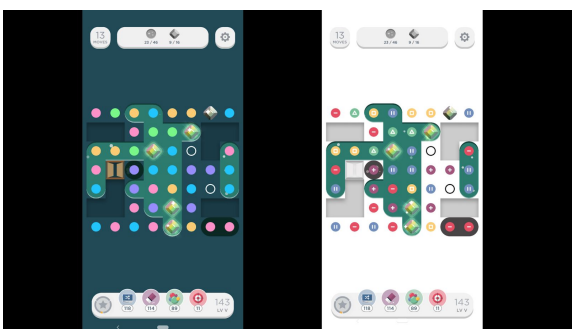

Figure 7. Before and after images of the mobile game application Two Dots with (right) and without (left) colorblind mode.

\section{Image Set 3}

The third set of images consisted of 3 screenshots of online web interfaces (see one example in Figure 8). These images were used to examine what elements on a web interface stand out to the red-green color deficient interviewee and why those specific elements stood out.

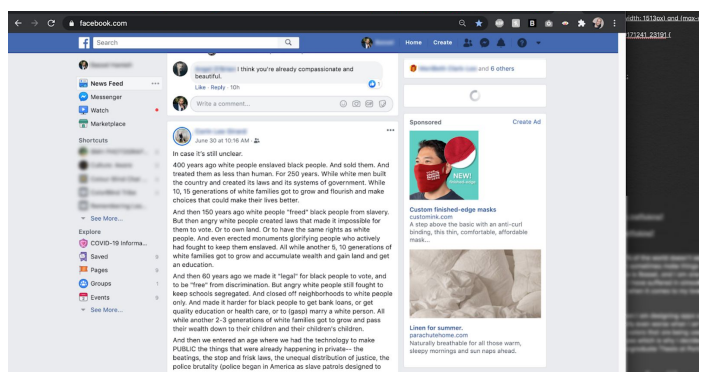

Figure 8. Facebook interface with mostly neutral colors other than the blue bar at the top and the image with bright red mask to the right.

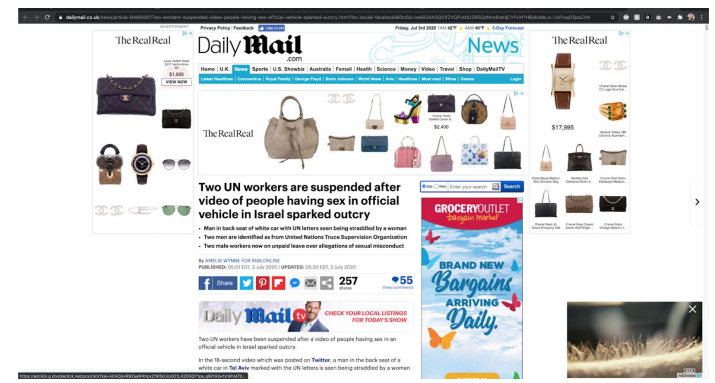

Figure 9. Daily Mail web interface with overcrowded visuals and colors with a mix of advertisements, headlines, and imagery.

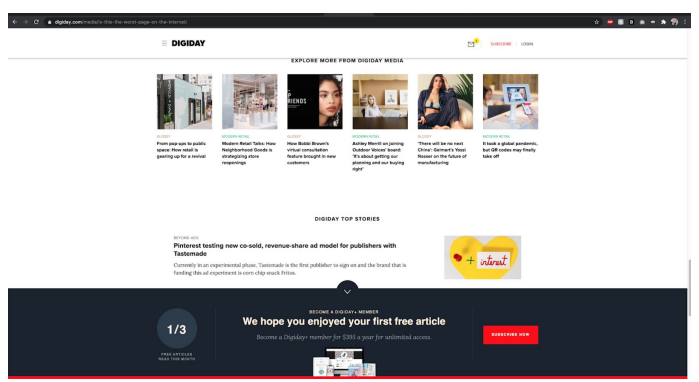

Figure 10. Bigday interface with a neutral color pallet and a couple of bright elements and imagery throughout the page.

\section{Qualitative Data Analysis}

Upon completion of the interviews, each interview was transcribed and I used a tagging process in the ATLAS.ti Qualitative Data Analysis Platform to identify common themes. From the initial themes surfaced, I narrowed down to a list of 5 key findings that resonated across the interviews.

\section{FINDINGS}

\section{Colorblindness Varies}

People with red-green color deficiency experience colors differently from the rest of the non-deficient world but also differently from one another. Not only can some be more green deficient or red deficient, meaning they do not see green, red, or both as well as other colors, but they can have those deficiencies in varying amounts of severity. An interviewee who is weak on the red side of the spectrum shared an experience which illustrates their deficiency when they said:
"A few years ago at my office, I was using an old CRT monitor, where one of the pins was defective on the monitor cable and I was operating without a red channel entirely. I didn't notice it for apparently a couple of months until somebody came by and asked why I had been running without red for so long."

Another interviewee who is sits on the green deficient side of the spectrum also shared their experience perceiving the color when they said:

"Green is probably a prettier color to other people, than it is to me. So, I think a lot of websites that use green or displays that are green based are probably just uglier looking to me in general."

And a third interviewee said:

"Certainly it varies individual to individual. My brother is also colorblind, and he's even more significantly red deficient than I am, he's almost entirely red blind. We didn't know that until we actually went to the Seattle Science Museum and they had a colorblindness and color deficiency exhibit on display and most of the Ishihara tests, neither of us could see anything, but there were one or two that I could perceive that he couldn't."

In addition to the quotes above which illustrated the variety of experiences red-green color deficient individuals have when it comes to color perception, interviewees' reactions to the mobile interface slides shown in Figures 4, 5, and 6 further underscored this variation. When asked to choose a button to "hang up" a phone call, interviewees described using a variety of methods to figure out how to decline the call. 1 out of the 4 participants couldn't see the red button and therefore relied on the icons and a process of elimination since they knew the other button was green. 2 out of the 4 participants were able to easily differentiate the red button from the green and chose it right away, and when the icons were taken off of the interface such as in 
Figure 4, only 1 out of the 4 participants had a hard time making a decision because they perceived red and green as the same color and therefore had to rely on their muscle memory.

Based on the quotes above and answers from the slide portion of the interviews, it is clear that the one-size-fits-all solutions for colorblindness outlined in the "Related Works" section are not going to work for the entire red-green color deficient population.

\section{Creative Work Arounds}

While interviewing the participants, all of them mentioned that they discovered being colorblind by accident. Some found out in their early lives as children or kids, illustrated through the following quote:

"I was in first grade and I was drawing a picture and it was a landscape. I was going to do the grass, so I went to the crayon drawer. And I did not know that this wasn't normal at the time, but I used to always check the label so I know whether it was green or brown. But this crayon had the label worn off of it. So, I just grabbed it, thinking it was green, but it was brown. And I finished coloring all the grass and turned the thing in and it didn't look wrong to me. And [my teacher] was like, "Yeah, you're probably colorblind."”

And others discovered their deficiency later in their life as teenagers, shown in the quote below:

"It was freshman year of high school, in biology
class. We're talking about genetics, one of the
examples that our teacher used, was for being
colorblind. So she threw up a colorblind test on
the big screen projector, and I was sitting in the
back of the class kind of freaking out, because I
couldn't see any of the numbers."

According to some of the responses from the interviews, living with a deficiency such as red-green colorblindness resulted in some creative coping strategies adopted by some of the participants. One of the interviewees said:

"I have noticed that if I wear a certain shade of sunglasses, I can tell the differences between different things sometimes."

Another interviewee used photo editing software to help differentiate between colors in images and graphs at work. They said:

"In Photoshop, I'll often work in the grayscale mode just so that I can see the grayscale indication. Then by toggling between the grayscale channels, I'll have an idea for what should be the RGB equivalent and alter back to $R G B$ for final publication and whatnot."

Two of the participants relied less on color differentiation and more on text and labeling. One of them said:
"I have a lot of labels and cues and written tips for [...] data presentation charts, as opposed to just having colors and having a legend on the side, especially when there are a lot of colors."

The other participant said:

"I don't think I really have a struggle in using interfaces, and it's because I don't rely on the colors. I rely on finding the texts as my primary helper or indicator of what I should be doing. "

Another participant relies on vocal communication to address issues which may arise from their colorblindness as they said, "I usually try to be really vocal with people, that's a strategy, I guess. If there's a situation where we're going to have to be communicating about colors."

While there are many technological solutions to help with red-green colorblindness which involve color differentiation, their unitary approaches did not meet the needs of the interviewees, resulting in the alternative approaches outlined above.

3. Colorblind People Understand Color Symbolism Most people who are colorblind aren't totally colorblind unless they have achromatopsia. This means that red-green colorblind individuals are still able to see colors, and most of the time red and green as well, but with different degrees of deficiency. This was illustrated while conducting the interview questions associated with the slides in Figures 4, 5 , and 6 . Three out of the 4 participants were able to see and differentiate the green and red buttons in the mobile interface as they identified them by color correctly. What was also observed is that the participants had ideas of color symbolism associated with the colors red and green based on their answers to some of the interview questions.

When asked about what the participants associate with the color red, all of them had the same associations. One participant said:

"I consider red to be dramatic and urgent. It's associated with stop and anything that's dangerous, as well. It's a lot of power and something absolutely important, critical."

Another said "Generally speaking, warning, stop, or halt. Something temperature-related like hot or warm." And when talking about interfaces, another participant mentioned, "I would consider it as a warning or something about caution or something that needs my attention on a website."

When asked about what the participants associate with the color green, most of them had the same associations as well. One participant said:

"Generally, [green is] a call to action. It's a way to proceed. Or that something is in a good state, you've completed an action, and you're ready to go forward with it." 
Another said that green is usually associated with "indicator contexts", meaning "things are okay are good to go or in a nominal state, they're safe or ready or something to that effect."

The idea that colorblind people still had symbolisms associated with color was even further proved during the mobile interfaces portion of the interviews. When asked why they chose the red button to hang up the phone call, one of the interviewees said "The color red I associate with hanging up..." and when they were asked why they wouldn't choose the other button, they said it was because "the right [button] appears green and [they] consider green as go or yes or answer phone."

Thus, in summary, while colorblind people may not see red or green in 'the same' way as non-colorblind people, most still do see red and green in their own way and associate meaning with these perceptions.

\section{Charts, graphs, and patterns are very difficult}

Through interviewing each of the participants, a trend was emerging when it came to their most difficult times of color differentiation while using and viewing web interfaces. Three out of the four participants mentioned that looking at graphs and charts that used numerous colors, including red and green, are very difficult to read; and all of the participants said that interfacts and images with lots of overlapping patterns are difficult to look at. One participant said:

"I've been looking at a lot of coronavirus graphs and stocks and so on. So whenever I view quantitative graphs, especially when they involve three or more colors, it becomes very hard to tell the different reds and greens that get used. And I don't think it's just the primary red versus primary green, but obviously, red mixed with other colors, it gets very hard for me to tell... I think also density maps. Anything which is a lot of data driven stuff on websites."

Another interviewee said:

"If there are elements that are mixing shades of red and green, especially in patterns, that's where I wouldn't quite be able to tell what the intended pattern is. I probably just see some strange red average."

And another also said, "If a website display is really busy with a lot of different stuff going on, a lot of times that's where the issue comes up for me."

Putting this in context with the third finding, we can conclude that while it is not necessary to totally eliminate the colors red and green to make interfaces accessible, keeping them may be important. It is still imperative to be thoughtful of how close together these colors appear and in general how cluttered and busy a composition is.

\section{Current accessibility modifications alter the user experience}

Discussing which colorblind tools the interviewees use to help with color differentiation prompted some very passionate responses. Especially when it came to how they affect the usability of the interfaces they use on a day to day basis. Since the severity of red-green colorblindness varies from one person to another based on the degree of deficiency of each of the colors red and green, their experiences using colorblindness tools also varies.

All of the interviewees had complaints about the change in appearance of the "color correcting" tools placed on their interfaces. Some said that they made the interfaces look less interesting, some said that they made them too harsh to look at, and others said they are better able to tell the colors apart. One of the participants expressed their disappointment when discussing the colorblind modes provided for some of the video games they play, saying "[The games] had a few colorblind modes. One of them was too harsh and one of them wasn't quite enough for me..." and another interviewee said "What I don't like [is that the colorblind modes] makes [the game] a lot duller... it kind of takes that experience away."

On the other hand, another interview seemed to take a more positive outlook, saying:
"The daltonising approach definitely changes the appearance, you end up losing color fidelity in the end because you're overlaying blue, so you basically just drop the pieces that used to be red. But what you gain by that is the differential mode... and you can see where there's supposed to be information. It takes longer to kind of interpret because you have to kind of mentally map it back in your head to what the presenter intended, but it's a way to determine where you're not perceiving something correctly."

And when it was time to look at some screenshots from a mobile game application, one shown in Figures 7, there also was a wide variety of opinions on the before and after colorblind mode images. One of the participants expressed their preference for the colorblind mode when they said, "I like the right one, the colorblind mode better because I can differentiate between the colors better."

Two of the participants expressed their dislikes of the colorblind mode. One of them said: "I think the user interface might be a little bit jarring at first to someone that is expecting a beautifully aesthetic color scheme." And the other said "My first initial thought, for this at least, is that I think the colorblind mode almost seems too cluttered when you're playing."

And another said that the colors in the none-colorblind mode version were easier to see, seen in the following quote: "Obviously I'm not seeing the colors correctly, but I still think [that] the other colors are more vivid when it's not on colorblind mode." 
Looking through all of the interview sections about color blindness tools and aids, it can be concluded that current coloblind modes have a significant impact on people's experience using interfaces, whether it being positive or negative.

\section{IMPLICATIONS FOR DESIGN}

\section{Interfaces should retain meaningful colors}

Though red-green color deficient individuals do not see red and green the same way non-deficient people see them, they still see some form of those colors that they have defined as red and green throughout their lives. Due to this and the findings above, it is now clear that color deficient people have the same symbolisms and associations to those colors that none-colorblind people have. Therefore, removing or replacing red and green from interfaces as a way to make them more accessible will make the interfaces even harder to interpret, as we are stripping away a layer of information which comes with the associations of the colors red and green. Instead, in the cases where the two colors are hard to see or differentiate from one another or individually, it is better to find ways to inform the user what those colors are, in order to maintain meaning and symbolism associated with the colors and interfaces.

\section{Consistency is critical}

Consistency in design and positioning of features within interfaces play a great role in how accessible they are. Due to muscle memory and mental mapping, red-green color deficient people rely less on the colors they see and more on their habits when it comes to interacting with familiar interfaces, even with colors that they cannot differentiate. One example which a participant mentioned about their experience with horizontal traffic lights versus the usual vertical ones illustrated this point well as they say:

"It turns out that my eyes are now conditioned by the position on the street light about the center versus the top versus the bottom. And when they were arranged horizontally, it was just very hard for me to be like, "Oh, is that red? Is that orange?" And many times, I thought of it as orange when it really was a yellow or was red."

And another participant while discussing why they chose the right button to decline the call using the mobile interfaces slides in Figures 4, 5, and 6 said:

\footnotetext{
"Because it's the right side. And I think on my phone it actually says accept [on the left] or decline [on the right], and so I've kind of just gotten to be used to.. I think that's more of a habit."
}

Therefore, when those familiar patterns are restructured and changed, it becomes very difficult for the red-green deficient individuals to rely on habit, especially without the ability to differentiate between colors and are forced to look for other ways to help them navigate interfaces such as iconography or the use of colorblindness tools.

\section{Customization is ideal}

The idea of control over the colors used within an interface was a unanimous desire across all of the participants. Each and every one mentioned having the ability to customize the colors they see when asked what an ideal colors blindness tool means to them. This is an important feature as each individual has their own reasons for why they would want to modify colors and customization is the antonym of a one-size-fits-all solution, which we have seen over and over again in the colorblind world.

Some colorblind modes as we discussed in the findings section are too harsh and some are too dull, some people prefer aesthetics over functionality, and some prefer functionality over aesthetics, and some who can't see a certain shade of red and green might want to replace it with a color with similar symbolic associations for better color differentiation. An interviewee's response when discussing the color customization features in a video game called Overwatch, illustrates the above well. They said:
"Overwatch is the only game I play that allows you to customize every color...Now that you can set all those colors manually, I can make enemies bright yellow instead of red. And my teammates are like light blue instead of green. I can just easily tell everything [apart]. But before that, it did cause some difficulty. [Otherwise] I would have to use the colorblind mode that was bright purple and it was just super, duper... It was clearly intended for someone who is very severely colorblind... the ideal for video games and websites and apps, is [one that has] an option to just let people manually set their colors to whatever they want."

Another participant discussed the ability to choose a different color than green when they said:
"Most interfaces use green, so that association is definitely there. But if I got to pick the button for instance, I would make it light blue."

One participant also suggested a standard for customization as well when they said:
"I feel like this should just be a standard where you have a tab or something in the top right or whatever it needs to be, that allows you to just customize the colors. And I think, honestly a lot of people would just do that, even if they weren't colorblind. People would take advantage of that. And it might even increase their mental investment in your website or whatever. I know that probably some visual designers would be horrified at the idea..."

In addition, one participant talked about his vision for more colorblind friendly world as they said: 


\begin{abstract}
"I would say, I would like to see a world in which websites can toggle between various color schemes to convey the same content to people who see colors differently, but being able to convey the same content. Maybe a person sees blue and yellow much better than they see red and green, and they kind of convey the same information and contrast..."
\end{abstract}

The general consensus from the interviewees reduced down to the fact that people know what they want and therefore should have the ability to manipulate the interfaces they use. A quote from a participant illustrates this very well when they say:

\begin{abstract}
"But the truth is, people know what looks good to them better than I think the designer is. I just, I feel like that's the true pinnacle of fixing color blindness or whatever, for those websites and pages. Just allow people to change everything. The text color, the background color, the panels, whatever you have."
\end{abstract}

\section{CONCLUSION}

Throughout time, red and green have become channels in which the world relies on to communicate. Whether it is in person or digital interfaces, red and green have become colors which contain strong symbolic associations. Red is known to grab attention, stop actions, and warn while green is known, generally for the opposite. The prominence of these symbolic associations which resulted in the widespread use and adoption of the two colors, have affected the red-green color deficient communities. In order to understand how these two colors are perceived by red-green colorblind individuals, I conducted interviews.

Once synthesized, I found that colorblindness varies from red-green deficient person to another and that no one solution is going to work for everyone. This resulted in some creative work-arounds which the interviewees had to embrace in order to see and communicate better. The most important finding is that it is now clear that colorblind people do understand color symbolism just as non-colorblind people do. In terms of struggles, almost all participants agreed that charts, graphs, and patterns are very difficult and most of them had complaints about current accessibility modifications, which seemed to alter the user experience either too much or too little.

Based on all of the research that I conducted and the interviews that I held, some implications emerged for design which would help. Due to the fact that red-green color deficient people still understood the symbolisms associated with red and green, it means that they can still perceive intentions behind colors within design, even though they are not able to see them. Due to the participants relying on muscle memory and habit, it became clear that consistency is critical. And lastly, all participants wished that they had the ability and full control to pick and change the colors they see in the interfaces they use. Which concludes that a one-size-fits-all solution is not a good fit and that customization will be the most effective ability to have when navigating web and application interfaces.

\section{ACKNOWLEDGMENTS}

I wish to express my sincere appreciation and deepest thank you to my honors thesis advisor, Doctor Ellie Harmon, who has been an incredible amount of support and guidance, and has encouraged me to be professional and do the right thing at the right time even when the road got tough. Without her persistent help, incredible research experience, and invaluable expertise, the goal of this project would not have been realized. I would also like to thank the interviewees who willingly gave up time out of their day to help with my undergraduate thesis which I am forever grateful for. Lastly, I would like to thank the honors program and all of its faculty at Portland State University, which have provided me an incredible undergraduate experience and made a better human and scholar.

\section{REFERENCES}

[1] Accessibility Color Wheel: https://www.giacomo.page/en/colorwheel/wheel.php. Accessed: 2020-08-12.

[2] Birch, J. 2012. Worldwide prevalence of red-green color deficiency. JOSA A. 29, 3 (Mar. 2012), 313-320. DOI:https://doi.org/10.1364/JOSAA.29.000313.

[3] Color meaning and symbolism: How to use the power of color: 2020.

https://www.canva.com/learn/color-meanings-symboli sm/. Accessed: 2020-07-17.

[4] Handbook of Color Psychology: 2015. /core/books/handbook-of-color-psychology/5A29A2BB A251510F1DCB9CBB746EE7D5. Accessed: 2020-07-17.

[5] Hillier, L. 2018. Considering colour blindness in UX design (with five examples). Econsultancy.

[6] Jefferson, L. and Harvey, R. 2007. An interface to support color blind computer users. Proceedings of the SIGCHI Conference on Human Factors in Computing Systems (San Jose, California, USA, Apr. 2007), 1535-1538.

[7] Madden, T.J., Hewett, K. and Roth, M.S. 2000. Managing Images in Different Cultures: A Cross-National Study of Color Meanings and Preferences. Journal of International Marketing. 8, 4 (2000), 90-107.

[8] Ribeiro, M. and Gomes, A.J.P. 2019. Recoloring Algorithms for Colorblind People: A Survey. ACM Computing Surveys. 52, 4 (Aug. 2019), 72:1-72:37. DOI:https://doi.org/10.1145/3329118.

[9] Spotlight on Cobalt Green / Winsor \& Newton: https://www.winsornewton.com/na/articles/colours/sp otlight-on-cobalt-green/. Accessed: 2020-07-17.

[10] Tanuwidjaja, E., Huynh, D., Koa, K., Nguyen, C., Shao, C., Torbett, P., Emmenegger, C. and Weibel, N. 2014. Chroma: a wearable augmented-reality solution for color blindness. Proceedings of the 2014 ACM 
International Joint Conference on Pervasive and Ubiquitous Computing (Seattle, Washington, Sep. 2014), 799-810.

[11] Understanding Success Criterion 1.4.1: Use of Color: https://www.w3.org/WAI/WCAG21/Understanding/use -of-color.html. Accessed: 2020-07-19.

[12] Understanding Success Criterion 1.4.3: Contrast (Minimum):

https://www.w3.org/WAI/WCAG21/Understanding/con trast-minimum. Accessed: 2020-07-17.

[13] Understanding WCAG 2.0: https://www.w3.org/TR/UNDERSTANDING-WCAG20 /Overview.html\#contents. Accessed: 2020-07-17.

[14] Using Red and Green in UI Design: 2019. https://uxplanet.org/using-red-and-green-in-ui-design66b39e13de91. Accessed: 2020-07-17.

[15] WAVE Web Accessibility Evaluation Tool: https://wave.webaim.org/. Accessed: 2020-08-12.

[16] Web Content Accessibility Guidelines (WCAG) 2.1: https://www.w3.org/TR/WCAG21/. Accessed: 2020-07-17. 